\title{
Nachwuchs-Förderungskampagne „Komm kleben!“
}

Um den Bedarf an Fachkräften auch in Zukunft trotz abnehmender Zahl der Schulabgänger decken zu können, hat die deutsche Klebstoffindustrie jetzt eine Online-Initiative gestartet.

D e deutsche Klebstoffindustrie gilt als weltweit führend auf dem Gebiet der Forschung und Entwicklung innovativer und leistungsfähiger Klebstoffsysteme. Zu verdanken hat die Industrie diese Spitzenposition auch ihren 13.253 technischen und kaufmännischen Mitarbeiter-/innen, die im Bereich der Forschung \& Entwicklung, der Produktion, der Anwendungstechnik bzw. im Marketing \& Vertrieb der deutschen Klebstoffunternehmen tätig sind.

Als hoch innovativer und wachstumsstarker Industriezweig hat die
Branche auch in Zukunft einen Bedarf an Fachkräften, deren Zahl allerdings - demografisch bedingt deutlich abnimmt. In weniger als acht Jahren wird sich die Zahl der Schulabgänger in Deutschland um über 20 Prozent verringern. Die deutsche Klebstoffindustrie hat daher die Nachwuchsförderungs-Kampagne „Komm kleben!“ ins Leben gerufen, mit der primär Schul- und Hochschulabsolventen, aber auch bereits ausgebildete Fachkräfte gezielt auf die vielfältigen beruflichen Perspektiven in der

Klebstoffindustrie aufmerksam gemacht werden sollen.

Die Webseite www.komm-kleben.de dient dabei sowohl als KommunikationsPlattform als auch als Job- \& Ausbildungsportal für Berufseinsteiger. Diese Internet-Plattform informiert über Ausbildungsmöglichkeiten, Jobprofile, offene Stellen, und sie gibt Hintergrundinformationen zur Branche und den einzelnen Klebstoffunternehmen.

Als weiteres Element bedient sich die Kampagne der sozialen Netzwerke, wie z. B. Facebook.

\section{Reis Technologie gewinnt Spe Award 2012}

Beim diesjährigen Wettbewerb der renommierten amerikanischen Vereinigung SPE (Society of Plastics Engineers) errang die Reis-Gruppe mit ihrer Anlage zur direkten Extrusion einer Schlauchdichtung den 1. Platz in der Kategorie Prozesstechnologien. Am Erfolg beteiligt waren Reis Extrusion, Reis Robotics (USA) und Reis Robotics (D), die das internationale Projekt unter Einbindung von weltweit führender Prozesstechnologie realisierten.

$\mathrm{D}$ er Einsatz dieses preisgekürten direkten Roboterextrusionsverfahrens ermöglicht im Vergleich zur herkömmlichen TSR-(EPDM)Profilmontage signifikante Einsparungen. Die Extrusion eines Vollprofils oder Mehrkammerprofils aus thermoplastischen Elastomeren (TPV) auf verschiedene Substrate kann in X-Y-ZRichtung erfolgen - und zwar ohne Vorbehandlung auf PP-Substraten.

Ferner sind dank Einsatz eines 6-achsigen Robotersystems eine hohe
Prozessflexibilität und Freiheiten beim Design gewährleistet. Hinzu kommt, dass sich bei diesem vollautomatischen Prozess das Nachbearbeiten erübrigt. Es fällt nahezu kein TPV-Abfall an, da überschüssiges Material dem Prozess wieder zugeführt wird.

Erwähnenswert sind schließlich die Dimensionsstabilität, die Oberflächengüte und nicht zuletzt die gute Recyclingfähigkeit.

Weitere Infos: www.reisrobotics.de
Die von Reis entwickelte Anlage zur direkten Extrusion von Schlauchdichtungen ermöglicht im Vergleich zur herkömmlichen TSR-(EPDM) Profilmontage deutliche Einsparungen.

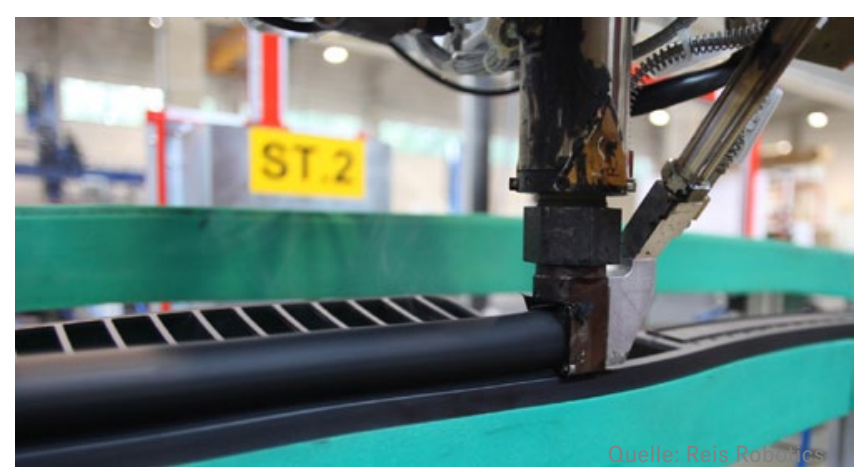

\section{Byk Additives \& Instruments investiert in Nordamerika}

uf dem kürzlich zugekauften A Werksgelände von Byk USA in Wallingford, Connecticut, entsteht derzeit eines neues 3-stöckiges Produktionsgebäude, das im Jahre 2014 fertiggestellt werden soll.

„Dieses Investment in Wallingford unterstreicht einmal mehr unser Ziel, im nordamerikanischen Markt $\mathrm{zu}$ wachsen und so viele Produkte wie möglich vor Ort zu produzieren“, so Dr. Roland Peter, Geschäftsbereichsleiter BYK Additives \& Instruments.

Das rund 36 Millionen Euro teure Gesamtprojekt stelle einen wichtigen Meilenstein in der weltweiten Unternehmensstrategie von BYK Additives \& Instruments dar und sei zudem das bisher größte Investment der gesamten Altana Gruppe außerhalb von Deutschland. 